\title{
Capsule Commentary on Paul et al., Size Misperception Among Overweight and Obese Families
}

\author{
Tiffany M. Powell-Wiley, MD, MPH \\ Cardiovascular and Pulmonary Branch, Division of Intramural Research, National Heart, Lung, and Blood Institute, National Institutes of Health, \\ Bethesda, MD, USA.
}

J Gen Intern Med 30(1):95

DOI: $10.1007 / \mathrm{s} 11606-014-3040-5$

(c) Society of General Internal Medicine 2014

$\mathrm{W}$ eight misperception, or failure to recognize the presence of overweight status or obesity, is recognized as a barrier to interventions promoting improved nutrition and physical activity, and as a potential contributor to the childhood obesity epidemic. ${ }^{1}$ Recent data from the National Health and Nutrition Examination Survey 2005-2012 suggest that weight misperception is highly prevalent among children/ adolescents, with more than two-thirds of overweight and almost one-half of obese U.S. children/adolescents aged 815 believing they were the right weight. ${ }^{2}$ However, little is understood about intergenerational weight perceptions within at-risk families, particularly when considering children's perceptions of their parents' weight.

Paul et. al. examined the relationship between weight status and 1) self-awareness of body size; 2) perceptions of body size across generations (child's perceptions of mother and vice versa); and 3) body size dissatisfaction in a cross-sectional study of predominantly Hispanic mother-child pairs recruited from an urban university clinic. ${ }^{3}$ One particularly novel finding is that children in the cohort often misclassified the weight status of their mothers, particularly when their mothers were overweight or obese. This finding suggests that a child's weight misperception may not be limited to his or her own body size, but may extend to misperceptions about family members and may be passed intergenerationally. As children misperceive obesity as normal weight for themselves and their mothers, they may be more tolerant of greater weight or weight gain over time. These findings suggest weight misperception as a potential mechanism by which obesity "spreads" across social networks. ${ }^{4}$

While information on prevalent cardiovascular risk factors among the mothers was provided, there were no data available on health beliefs in the study population. It would be interest- ing to see whether attitudes about overall health status and risk for obesity-related chronic diseases varied based on body-size perceptions among the mothers and/or children. Prior work suggests that obese adults with weight misperception also underestimate the risk of obesity-associated comorbidities, including diabetes, hypertension, or cardiovascular events. ${ }^{5}$ Overall, this study reinforces the need to develop interventions that acknowledge and address cultural standards of weight for diverse populations if we are to stem the tide of obesity in the United States.

Conflict of Interest: The author has no conflict with any of the material in this manuscript. Funding support for the author is provided by the Division of Intramural Research of the National Heart, Lung, and Blood Institute (NHLBI) at the National Institutes of Health (NIH).

Corresponding Author: Tiffany M. Powell-Wiley, MD, MPH; Cardiovascular and Pulmonary Branch, Division of Intramural Research, National Heart, Lung, and Blood InstituteNational Institutes of Health, Bethesda, MD 20892, USA (e-mail: Tiffany.powell@nih.gov).

\section{REFERENCES}

1. Chen HY, Lemon SC, Pagoto SL, Barton BA, Lapane KL, Goldberg RJ. Personal and parental weight misperception and self-reported attempted weight loss in US children and adolescents, national health and nutrition examination survey, 2007-2008 and 2009-2010. Prevent Chron Dis. 2014;11:E132. doi:10.5888/pcd11.140123.

2. Sarafrazi N, Hughes JP, Borrud L, Burt V, Paulose-Ram R. Perception of weight status in U.S. children and adolescents aged 8-15 years, 2005-2012. NCHS data brief. 2014;(158):1-8.

3. Paul TK, Sciacca RR, Bier M, Rodriguez J, Song S, Giardina EV. Size Misperception among overweight and obese families. J Gen Intern Med. 2014;(SPI: 3002).

4. Christakis NA, Fowler JH. The spread of obesity in a large social network over 32 years. N Engl J Med. 2007;357(4):370-9. doi:10.1056/ NEJMsa066082.

5. Powell TM, de Lemos JA, Banks K, Ayers CR, Rohatgi A, Khera A, et al. Body size misperception: a novel determinant in the obesity epidemic. Arch Intern Med. 2010;170(18):1695-7. doi:10.1001/ archinternmed.2010.314. 